\title{
High Rate Space Frequency Block Codes for Next Generation 802.11 WLANs
}

\author{
Lei Shao*, Sumeet Sandhu ${ }^{\dagger}$, Sumit Roy* and Minnie $\mathrm{Ho}^{\dagger}$ \\ ${ }^{*}$ Dept. of Electrical Engineering, University of Washington, Seattle, WA 98195-2500 \\ Email: $\{1 \mathrm{~s} 7$, roy $\} @$ ee.washington.edu \\ $\dagger$ Intel Corporation, M/S RNB-6-49, 2200 Mission College Blvd, Santa Clara, CA 95052 \\ Email: \{sumeet.sandhu,minnie.ho\}@intel.com
}

\begin{abstract}
Achieving higher link rates in support of newer services and increasing range are the two main goals for next generation wireless LAN networks. This work primarily contributes to a high rate solution, based on a novel extension to the rate-1 space-frequency block code (SFBC) design in [1] to full rate case. To further improve performance without increasing complexity, we propose unequal power allocation at the transmitter with no requirement of channel knowledge. Compared with standard 802.11a transmitter which uses convolutional code with a single transmit antenna and antenna selection diversity among 2 receive antennas with soft Viterbi decoding, we show that our HR-SFBC code with 2 transmit antenna not only doubles the throughput (as expected) but also provides additional link margin (and hence improves range).
\end{abstract}

\section{INTRODUCTION}

Current WLANs based on $802.11 \mathrm{~b}$ standard (commonly known as Wi-Fi) support a maximum link rate of $11 \mathrm{Mbps}$; this is insufficient for many emerging applications. The demand for higher bit rates naturally led to more spectrally efficient modulation schemes such as Orthogonal Frequency Division Multiplexing (OFDM) that forms the basis of the more recent .11a WLANs. OFDM is well-known to be robust to multipath fading that characterizes wireless channels. To further increase spectral efficiency, additional link layer innovations are needed. The currently active IEEE 802 High Throughput Task Group (802.11n) aims to increase peak rate well beyond $100 \mathrm{Mbps}$ for next generation WLANs. It is anticipated that the use of multiple antennas on both transmit and receive, i.e. multi-input, multi-output (MIMO) technology, that allows both (i) high-rate transmission without bandwidth increase and (ii) greater link reliability via the exploitation of the multipath diversity inherently available over the channel will be an integral component of next generation WLANs. Thus, a link layer based on a combination of MIMO and OFDM (MIMOOFDM ) appears particularly promising for achieving highthroughput.

MIMO designs for WLANs ideally should maximize link throughput and diversity gain with a low decoding complexity receiver; there does not exist as yet a single desirable solution that achieves all three simultaneously. The primary goal in this work is to achieves both high rate and high diversity of the channel in a single code design. Specifically, for MIMO systems with $N \geq M$, we design full rate codes that transmit $M$ uncoded symbols/ channel use, where $N$ and $M$ denote number of receive and transmit antenna, respectively.

High rate or 'spatial multiplexing' MIMO schemes have been proposed by [6][7][8] [9] [10] [11].In D-BLAST, layers are transmitted in a diagonal fashion. To maintain same diversity order for all layers, zeros has to be padded at the beginning and end of the frame. In addition, D-BLAST requires short but efficient diagonally layered coding schemes to reduce its boundary space-time edge wastage. Compared with DBLAST, V-BLAST is simpler and eliminates the need of short block codes. In V-BLAST, each independently coded layer is transmitted on one of the antennas, which causes different layers attain different diversity orders and the processing order of layers therefore becomes important. In order to reduce the effect of error propagation, an optimal ordering scheme was introduced in [7] at the price of significantly increased complexity. Moreover, both [6] and [7] need underlying low rate SISO codes to achieve diversity at the price of rate efficiency. [9] introduced a method that uses a design parameter to adjust number of precoded layers thus trade space-diversity gain for high rates (but not full rates) for any number of tranmsit and receive antennas in flat fading channel case. [8] is a block version of D-BLAST combined with linear constellation precoding across antennas, time slots and tones to collect space-frequency diversity for MIMO-OFDM. It cannot achieve full-rate because of the D-BLAST structure and has longer processing delay due to coding across several OFDM block times. More recently, [10] and [11] introduced full-rate, full-diversity schemes. However, they guarantee full diversity only if the component SISO codes for all layers are jointly detected. Although these two full-rate, full-diversity codes can be extended to frequency-selective fading channels for MIMOOFDM, their decoding complexity of $\left.O\left(\frac{N_{c}}{M L} \times\left(2 M^{2} L\right)^{3}\right)\right)$ based on sphere decoding (where $N_{c}$ is number of subcarriers in OFDM system and $L$ is number of independent taps for frequency selective fading channels) is significantly high. In contrast, our HRSFBC can be decoded with complexity $O\left(\frac{N_{c}}{K} \times(2 M K)^{3}+N_{c} M^{3}\right)$ with design parameter $K \ll L$.

Our previously proposed rate- $1 \mathrm{SFBC}[1]$ is a diversitymaximal code. However, the rate was low(i.e. only rate-1). In this work, we propose extensions to our earlier designed rate-1 SFBC[1] to full rate, i.e., we propose a new rate-M (for $M \leq N$ ) SFBC encoder/decoder. In order to achieve a desirable tradeoff between rate, performance and complexity, 
we only perform sphere decoding for each layer within a group (we call the diagonal positions used in rate-1 SFBC case one 'layer') and use suboptimal detection such as ZF-BLAST or MMSE-BLAST without ordering on each tone among layers.

As is known for BLAST in flat fading channels, optimal user ordering [7] can greatly improve performance at a price of significantly increased detection complexity. However, for full-rate SFBC, since precoded symbols from the same group within the same layer are distributed across multiple tones, frequency selective fading will cause the optimal user-ordering at the receiver to differ for various tones since the channel matrix for neighboring tones of a MIMO-OFDM can vary significantly. This will cause difficulty since the whole precoded layer must be detected correctly prior to cancellation. To make reliable detection without increasing decoding complexity, we propose a novel unequal power allocation scheme across layers (with no requirement of channel knowledge at the transmitter side) in which the layer with the largest transmit power is detected first. In summary, the major contributions of this paper are as follows: (1) design novel rate-M SFBC encoder $(N \geq M)$; (2)For reliable detection, utilize unequal power allocation across layers so that the layer with largest transmit power is detected before other layers using sphere-decoding. (3)MMSE-BLAST without ordering is implemented on a toneby-tone basis by first using generalized QR decomposition on equivalent channels so that nulling is completed at once; this is distinct from QR decomposition in ZF-BLAST type of detection[8] [10].

\section{High RATE SFBC}

\section{A. Code Construction of Rate-1 SFBC}

In our previously proposed rate-1 SFBC[1][5] (see. Fig. 4), uncoded symbol vectors were precoded by a square, linear, complex-field matrix without introducing redundancy as shown in Fig.1. The precoder is of size $M K \times M K$, where design parameter $K$ is an estimate of the channel taps $L$, and $K \geq L$ is required to collect maximum diversity gain offered by MIMO frequency-selective channels. These precoded symbols are distributed over uncorrelated tones and multiple antennas so as to maximize the diversity gain when (nearoptimal) sphere decoding[4][14] is applied at the receiver across these tones and antennas. While this code represented an advance in terms of achieving the maximum diversity, the rate was low (i.e. only rate-1).

\section{B. High Rate SFBC}

Compared with rate-1 SFBC in Figure.2, HR SFBC (see Fig.3 and Fig. 4) makes full use of all the positions in the space-frequency code matrix. Precoded symbols are mapped to the space-frequency code matrix in a row circular fashion similar to [10]; however, the difference is that our HRSFBC is in space-frequency domain with the block interleaving type mapping inherited from rate- $1 \mathrm{SFBC}$. If we denote the positions occupied by rate-1 SFBC a 'layer', HRSFBC has $M$ layers.

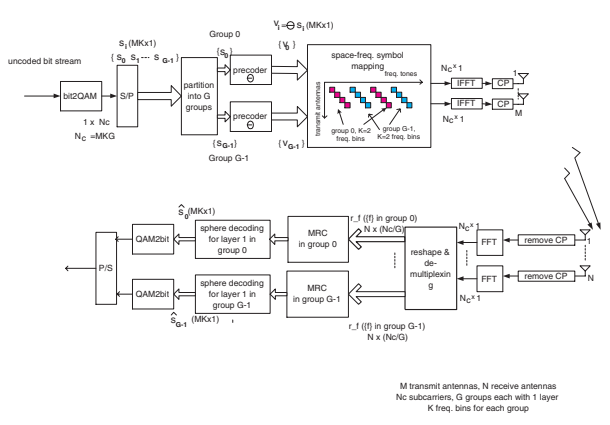

Fig. 1. System block diagram of rate-1 SFBC-OFDM

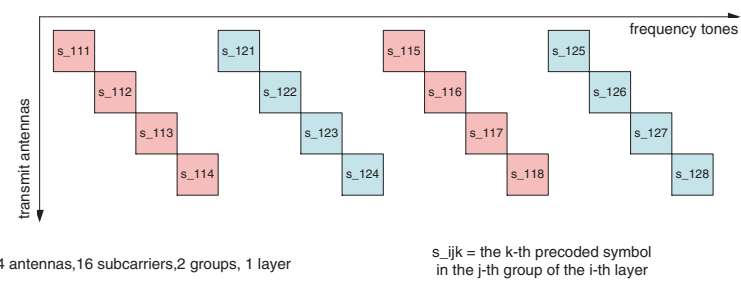

Fig. 2. space-frequency symbol mapping for rate-one space-frequency block $\operatorname{code}(\mathrm{K}=2)$

For unequal power allocated HRSFBC, after QAM modulation and LCF precoding, we multiply the precoded symbol vector corresponding to the $m$-th layer by coefficient $\sqrt{2^{m-1} M / \text { sum }}$ (where sum is defined as sum $=$ $\sum_{m=1}^{M} 2^{m-1}$ ), and then map the power-weighted vector to the $m$-th layer in the space-frequency code matrix using the same type of space-frequency symbol mapping. Assume that $E\left|s_{m}\right|^{2}=E_{s}, \quad$ for $m=1, \cdots, M$. Because

$\sum_{m=1}^{M} E\left|\sqrt{\frac{2^{(m-1)} M}{s u m}} s_{m}\right|^{2}=\sum_{m=1}^{M} M \frac{2^{(m-1)}}{s u m} E_{s}=\sum_{m=1}^{M} E\left|s_{m}\right|^{2}$

it is clear that the total transmit power is the same as in equalpower case. Moreover, no channel knowledge is required at the transmitter side.

\section{Space-Frequency Decoding of HRSFBC}

The received signal at the $f$-th tone $\left(f \in 0, \cdots,\left(N_{c}-\right.\right.$ 1)), and the $j$-th receive antenna can be written as $r_{j}(f)=$ $r_{j}((k-1) G M+g M+m-1)$, where $m \in 1, \cdots, M$ is the column index for each $M \times M$ sub-matrix, $k \in 1, \cdots, K$, $g \in 0, \cdots, G-1$. To reduce the decoding complexity, we only consider suboptimal detection methods in this paper so that

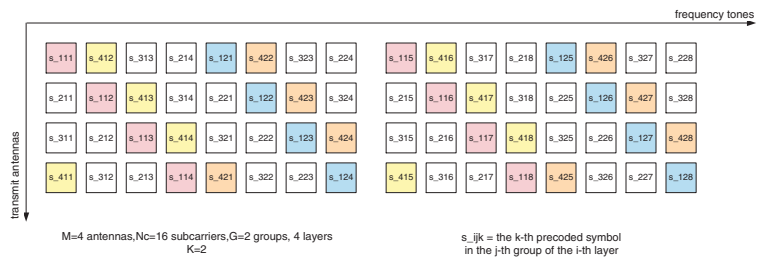

Fig. 3. space-frequency symbol mapping for high rate SFBC 


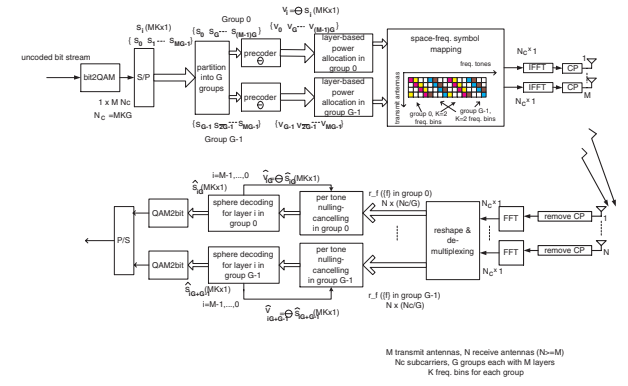

Fig. 4. System block diagram of HRSFBC-OFDM

the approximate decoding complexity is $O\left(\frac{N_{c}}{K} \times(2 M K)^{3}+\right.$ $N_{c} M^{3}$ ) from the average decoding complexity of sphere decoder. It is easy to show that the decoding complexity proposed is much lower than that of optimal decoding i.e. $O\left(\frac{N_{c}}{M L} \times\left(2 M^{2} L\right)^{3}\right)$ as in [10] .For HRSFBC, the diversity gain of the overall system is $M K(N-M+1)$ [15]. The detailed diversity analysis is omitted due to space limitation.

\section{A. ZF-BLAST type of detection with $Q R$ decomposition}

At the $f$-th tone, collecting received signals from $N(N \geq$ $M)$ receive antennae, we get

$$
\begin{aligned}
\mathbf{r}_{f} & =\left[\begin{array}{lll}
r_{1, f} & \cdots & r_{N, f}
\end{array}\right]^{T} \\
& =\mathbf{H}_{f} \mathbf{u}_{f}+\mathbf{w}_{f}
\end{aligned}
$$

where

$$
\mathbf{u}_{f}=\mathbf{P}_{m}\left[\begin{array}{c}
\theta_{(k-1) M+m}^{T} \mathbf{s}_{g} \\
\theta_{(k-1) M+m}^{T} \mathbf{s}_{G+g} \\
\vdots \\
\theta_{(k-1) M+m}^{T} \mathbf{s}_{(M-1) G+g}
\end{array}\right]
$$

with the $m$-th permutation matrix $P_{m}$ defined as

$$
\mathbf{P}_{m}=\left[\begin{array}{ll} 
& \mathbf{I}_{m-1} \\
\mathbf{I}_{M-m+1} &
\end{array}\right]
$$

Therefore,

$$
\mathbf{r}_{f}=\underbrace{\mathbf{H}_{f} \mathbf{P}_{m}}_{\mathbf{B}_{f}} \underbrace{\left[\begin{array}{c}
\theta_{(k-1) M+m}^{T} \mathbf{s}_{g} \\
\theta_{(k-1) M+m}^{T} \mathbf{s}_{G+g} \\
\vdots \\
\theta_{(k-1) M+m}^{T} \mathbf{s}_{(M-1) G+g}
\end{array}\right]}_{\mathbf{x}_{f}}+\mathbf{w}_{f}
$$

We then perform a $\mathrm{QR}$ decomposition on equivalent channel $\mathbf{B}_{f}$ to obtain $\mathbf{B}_{f}=\mathbf{Q}_{f} \mathbf{R}_{f}$, where $\mathbf{Q}_{f}$ is a unitary matrix and $\mathbf{R}_{f}$ is a upper triangular matrix. Left multiplying $\mathbf{r}_{f}$ with $\mathbf{Q}_{f}^{H}$ yields

$$
\begin{aligned}
\mathbf{y}_{f} & =\mathbf{Q}_{f}^{H} \mathbf{r}_{f} \\
& =\left[\begin{array}{ccc}
r_{1,1} & \cdots & r_{1, M} \\
& \ddots & \vdots \\
& & r_{M, M}
\end{array}\right] \underbrace{\left[\begin{array}{c}
\theta_{(k-1) M+m}^{T} \mathbf{s}_{g} \\
\theta_{(k-1) M+m}^{T} \mathbf{s}_{G+g} \\
\vdots \\
\theta_{(k-1) M+m}^{T} \mathbf{s}_{(M-1) G+g}
\end{array}\right]}_{\mathbf{x}_{f}} \\
& +\mathbf{Q}_{f}^{H} \mathbf{w}_{f}
\end{aligned}
$$

To recover the $M$ symbol vectors $\mathbf{s}_{(p-1) G+g}$ (each of length $M K$ ) for $p=M, \cdots, 1$ in the $g$-th group, we need to collect all information from $M K$ tones corresponding to the $g$-th group. We first recover the symbol vector with its $((k-1) M+$ $m$ )-th precoded element at the bottom of $\mathbf{x}_{f}$, i.e. $\mathbf{s}_{(M-1) G+g}$. In the following, we regard $\mathbf{y}_{f}$ as the equivalent received symbol vector for the $f$-th tone. We collect symbols at same equivalent receive antenna (starting from the last dimension) of $\mathbf{y}_{f}$ with $\{f\}$ chosen from $[M G(k-1)+(g-1) M+1]$ to $[M G(k-1)+g M]$, for $k=1, \cdots K$. If we use $\left\{z_{i}\right\}_{i=1}^{M K}$ to denote these $M K$ symbols, we get the following

$$
\begin{aligned}
{\left[\begin{array}{c}
z_{1} \\
z_{2} \\
\vdots \\
z_{M K}
\end{array}\right] } & =\left[\begin{array}{ccc}
r_{M, M, 1} & & \\
& \ddots & \\
& & r_{M, M, M K}
\end{array}\right] \\
& \times \Theta_{(M-1) G+g}+\left[\begin{array}{lll}
n_{1} & \cdots & n_{M K}
\end{array}\right]^{T}(6)
\end{aligned}
$$

where $r_{M, M, i}$ denotes the $r_{M, M}$ for the $i$-th tone in this length- $M K$ tone set and $n_{i}$ denotes the corresponding noise element.

Next, we propose sphere decoding (SD) [4][14] to recover $\hat{\mathbf{S}}_{(M-1) G+g}$ since it can achieve near-optimum performance(in the maximum likelihood sense) with polynomial complexity in $M K$ (e.g. $O\left((M K)^{3}\right)$ for $\Theta$ real) regardless of the constellation size ${ }^{1}$. We then regenerate $\Theta \hat{\mathbf{s}}_{(M-1) G+g}$ and cancel its interference on $\left\{\mathbf{y}_{f}\right\}$ with $\{f\}$ corresponding to the $M K$ tones in the $g$-th group. After that, we decode remaining $M-1$ precoded symbol vectors sequentially from the bottom to the top of $\left\{\mathbf{x}_{f}\right\}$ for the specific $g$ as described.

\section{B. MMSE-BLAST Type Detection with Generalized $Q R$ - Decomposition}

This represents a simple improvement on zero forcing (ZF) detection by noting that since $\mathbf{r}_{f}=\mathbf{B}_{f} \mathbf{x}_{f}+\mathbf{w}_{f}$, for each tone [c.f.(4)], the MMSE detector can be re-cast as follows

$$
\begin{aligned}
\hat{\mathbf{x}}_{f} & =\left(\frac{N_{o}}{E_{s}} \mathbf{I}_{M}+\mathbf{B}_{f}^{H} \mathbf{B}_{f}\right)^{-1} \mathbf{B}_{f}^{H} \mathbf{r}_{f} \\
& =[\underbrace{\left[\begin{array}{c}
\mathbf{B}_{f} \\
\sqrt{\frac{N_{o}}{E_{s}}} \mathbf{I}_{M}
\end{array}\right]}_{\mathbf{H}_{e f}}]^{\dagger}\left[\begin{array}{c}
\mathbf{r}_{f} \\
\mathbf{0}
\end{array}\right]
\end{aligned}
$$

\footnotetext{
${ }^{1}$ Since in our case, both $\Theta$ and $\mathbf{s}_{g}$ are complex, we write an $M K$ dimensional complex vector $\mathbf{y}_{g}$ as a $2 M K$-dimensional real vector.
} 
as per [13]. Regarding MMSE as generalized ZF, the above implies that MMSE or generalized ZF uses pseudo-inverse of equivalent channel matrix $\mathbf{H}_{e f}$ instead of $\mathbf{B}_{f}$. Thus, if we do $\mathrm{QR}$ decomposition on the equivalent channel matrix $\mathbf{H}_{e f}$, we have already considered the effect of noise in nulling. All other procedures are the same as those in ZF-BLAST type of detection with QR decomposition.

\section{MMSE-BLAST Type Detection for Unequal Power Alloca- tion On Transmit}

As is known, error propagation occurs in BLAST type detection per tone if the initially detected precoded symbol is incorrect. For reliable initial detection when symbols are precoded across tones, we can assign different powers to different 'layers' while keeping the total power the same as that in equal-power case. For example, we can geometrically increase power on layers with the last layer having the largest transmit power. For this case, in the equivalent channel matrix per tone $\mathbf{H}_{e f}$ for QR decomposition, $\mathbf{B}_{f}$ will be replaced by

$\mathbf{B}_{f, \text { unequal }}=\mathbf{B}_{f}\left[\begin{array}{lll}\sqrt{M / \text { sum }} & & \\ & \ddots & \\ & & \sqrt{2^{M-1} M / \text { sum }}\end{array}\right]$

(8)

where sum $=\sum_{i=1}^{M} 2^{i-1}$. All other processing is the same as that in Section B above.

\section{Simulation Results and Conclusions}

We conducted simulation experiments for both synthetic channels as well as channel data obtained from measurements. For the simulated channels, an $L$-tap frequency selective channel between each transmit-receive antenna pair with independent complex Gaussian coefficients with total power of unity was used. We used $L=2$ with i) uniform power delay and ii) exponential decay power delay profile with $\tau_{r m s}=50 \mathrm{~ns}$. The total average symbol energy on all $M$ transmit antennas was normalized to $E_{s}=1$. The power for different layers in unequal power HRSFBC case was allocated geometrically. For measured channels, the data set 14 [12](see Fig. 5) obtained from Intel Labs measurements was used. The indoor channel measurements were conducted at $5-6 \mathrm{GHz}$ band in the conference room with door closed. The MIMO measurements are comprised of several SISO measurements. All system parameters are the same as those of 802.11a standard. In order to make a fair comparison between schemes with/without convolutional coding, we fix the data rate/antenna. Moreover, we fix the packet size to be 1000 bytes to compare packet error rate (PER). It is worthy of note that PER will equal the outage probability for a specific signaling rate (outage capacity) if the transmitter does not know the channel. For this reason, the PER curve rather than BER is more important. For a specific scheme, the SNR value that we are particularly interested is the minimum SNR needed for $P E R \leq 0.1$. We use this value to compare schemes with the same signaling rate.

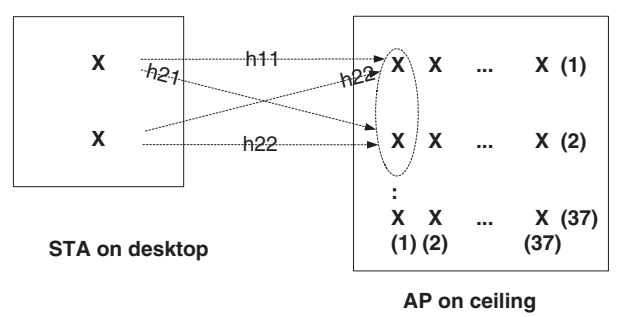

Fig. 5. measured channel set 14

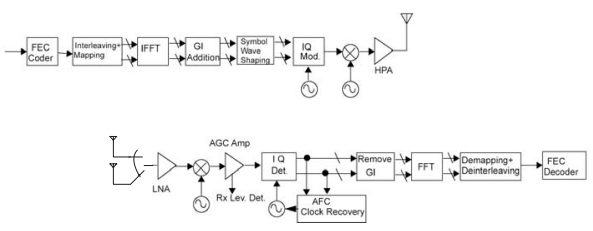

Fig. 6. baseline 802.11a with receive antenna selection and soft decoding

In addition, for baseline 802.11a system (see Fig.6), we use soft-decision decoding assuming perfect CSI at the receiver and the best (one of two) receive antenna based on received power (labelled "( $(2) 1 \times 1$ ”).

It is very clear from Fig. 7 that MMSE-BLAST type of detection offers better BER performance than ZF-BLAST type of detection. Moreover, unequal-power-allocated transmission scheme outperforms equal-power-allocated transmission scheme due to mitigation of error propagation.

(I) Throughput comparison

Fig. 8 and Fig. 9 shows throughput comparison between (un)equal-power-allocated HRSFBC and 802.11a with receive antenna selection and soft decoding in both simulated channel and measured channel. From the results, we find that by using 3 receive and 2 transmit antennae (labelled " $3 \times 2$ "), and setting design parameter $K=4$, both the unequal-powerallocated scheme and the equal-power-allocated scheme outperform 802.11a scheme (with receive antenna selection and soft decoding) at PER=0.1 while doubling the throughput (the BER advantage of HRSFBC is more significant from the plot). Note that since the system diversity gain is $M K(N-M+1)$, performance of HRSFBC can be much better if we increase

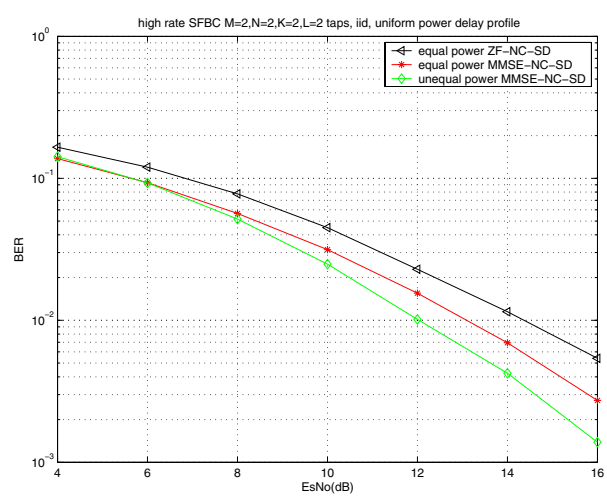

Fig. 7. performance comparison in simulated channels 


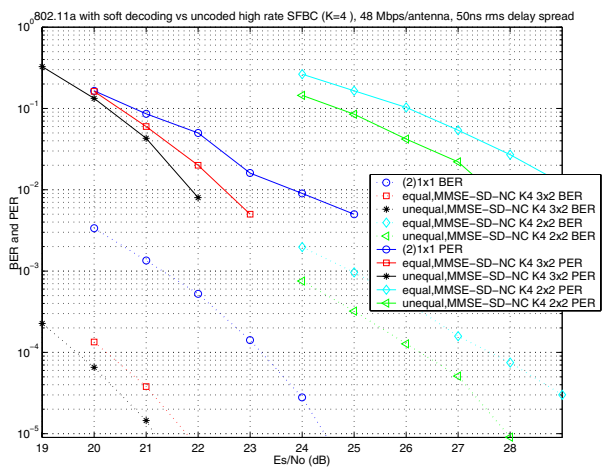

Fig. 8. throughput comparison in simulated channels

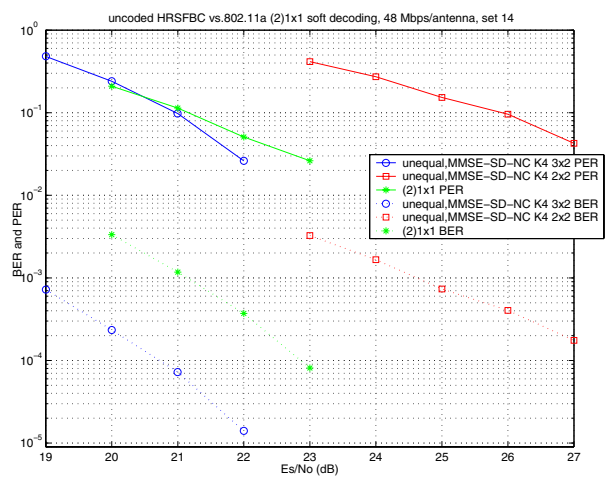

Fig. 9. throughput comparison in measured channels

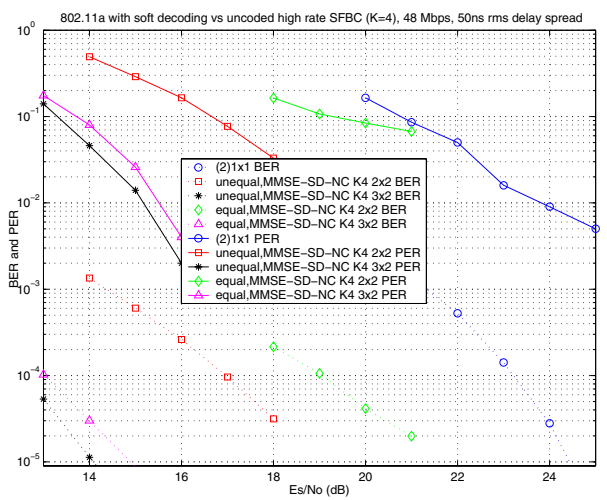

Fig. 10. range comparison in simulated channels

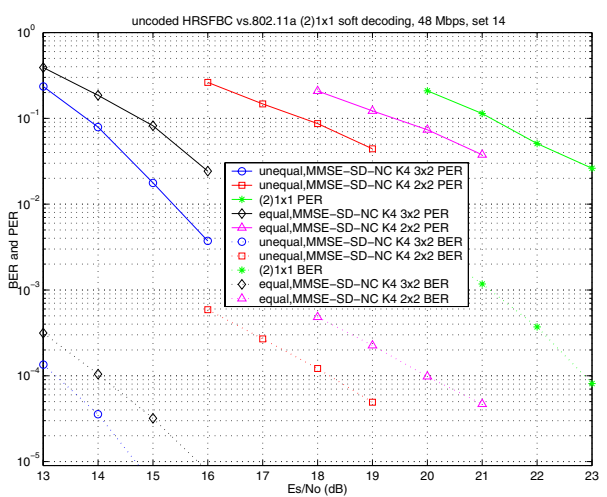

Fig. 11. range comparison in measured channels the value of design parameter $K$ or the number of receive antennae. It is shown that for this suboptimal detection of HRSFBC, increasing the number of receive antenna by just 1 can give us additional 4-5 $\mathrm{dB}$ gain.

(II) Range comparison

Fig.10 and Fig. 11 show range extension using HRSFBC in both simulated channel and measured channel. Since at $B E R=10^{-5}$, unequal-power-allocated HRSFBC with $K=$ 4 , $3 \times 2$ outperforms $802.11 \mathrm{a}$ by $10 \mathrm{~dB}$ and unequal-powerallocated HRSFBC with $K=4,2 \times 2$ outperforms 802.11a by $5.5 \mathrm{~dB}$; if we use pathloss exponent $r=3$, we get 2.15 times and 1.52 times range extension, respectively. For the same reason as mentioned in throughput comparison, much better performance can be expected with larger $K$ or larger number of receive antennae.

\section{REFERENCES}

[1] L. Shao, S. Roy and S. Sandhu, "Rate-one Space frequency block codes with maximum diversity gain for MIMO-OFDM," IEEE Global Telecommunications Conference, 2003.(GLOBECOM '03), Volume: 2 , 1-5 Dec.2003 Pages: 809 - 813.

[2] Y. Xin, Z. Wang and G. B. Giannakis, "Linear Unitary Precoders for Maximum Diversity Gains with multiple Transmit- and Receive-Antennas," Proc. of 34th Asilomar Conf. on Signals, Systems, and Computers, pp. 1553-1557, Pacific Grove, CA, Oct. 29-Nov. 1, 2000 (invited).

[3] Y. Xin, Z. Wang and G. B. Giannakis, " Space-Time ConstellationRotating Codes Maximizing Diversity and Coding Gains," Proc. of GLOBECOM, vol. 1, pp. 455-459, San Antonio, TX, Nov. 25-29, 2001.

[4] M. O. Damen, A. Chkeif, and J.-C. Belfiore, "Lattice codes decoder for space-time codes," IEEE Communications Letters, vol.4, no.5,pp.161163, May 2000.

[5] L. Shao and S. Roy, "Rate-one Space frequency block codes with maximum diversity gain for MIMO-OFDM," submitted to IEEE Trans. on Wireless Communications, May 2003.

[6] G. J. Foschini, "Layered space-time architecture for wireless communication in fading environment when using multi-element antennas," Bell Labs. Tech. Journal, vol.1, no.2, pp 41-59, Autumn 1996.

[7] P. W. Wolniansky, G. J. Foschini, G. D. Golden, and R. A. Valenzuela,"VBLAST:An architecture for realizing very high data rates over the richscattering wireless channels," Proc. of URSI International Symposium Signals, Systems, and Electronics, Italy, 29 Sept.-2 Oct. 1998. Pages: $295-300$.

[8] Y. Xin, and G. B. Giannakis, " High-Rate Space-Time Layered OFDM," IEEE Communications Letters, vol. 6, no. 5, pp. 187-189, May 2002.

[9] Y. Xin, Z. Liu, and G. B. Giannakis, " High-Rate Layered Space-Time Coding based on Constellation Precoding," Proc. of Wireless Communications and Networking Conf., vol. 1, pp. 471-476, Orlando, FL, March $17-21,2002$.

[10] X. Ma, and G. B. Giannakis, "Complex Field Coded MIMO Systems: Performance, Rate, and Tradeoffs," Wireless Communications and Mobile Computing, pp. 693-717, November 2002.

[11] H. El Gamal and M. O. Damen, "Universal space-time coding," IEEE Transactions on Information Theory, Volume: 49 , Issue: 5, May 2003 Pages:1097 - 1119

[12] S. Sandhu, M. Ho, C. Prettie, D. Cheung "Capacity analysis of indoor MIMO measurements at 5-6GHz," Intel internal document, Intel Corporation.

[13] B. Hassibi, "A fast square-root implementation for BLAST," Conference Record of the Thirty-Fourth Asilomar Conference on Signals, Systems and Computers, 2000. ,Volume: 2, 29 Oct.-1 Nov. 2000 Pages:1255 1259 vol. 2

[14] A. M. Chan, I. Lee, "A new reduced-complexity sphere decoder for multiple antenna systems," IEEE International Conference on Communications, 2002 (ICC 2002), Volume: 1, 28 April-2 May 2002 Pages:460 -464 .

[15] L.Shao, S. Sandhu, S. Roy, M. Ho,"High rate next generation 802.11 WLANs using space-frequency block codes," submitted to Special Issue on MIMO Commun., Wiley's Wireless Communications and Mobile Computing (WCMC), January 2004. 